\title{
Bubble, stripe, and ring phases in a two-dimensional cluster with competing interactions
}

\author{
K. Nelissen, ${ }^{*}$ B. Partoens, ${ }^{\dagger}$ and F. M. Peeters ${ }^{\ddagger}$ \\ Departement Fysica, Universiteit Antwerpen (Campus Middelheim), Groenenborgerlaan 171, B-2020 Antwerpen, Belgium
}

(Received 20 October 2004; revised manuscript received 28 February 2005; published 6 June 2005)

\begin{abstract}
A system of classical charged particles confined in a two-dimensional trap and interacting through a competing short-range attraction and long-range repulsion potential is studied. The structure of the system strongly depends on the interaction range of the short-range attraction potential and the total number of particles. Depending on the appropriate choice of parameters for the attractive potential, the particles organize themselves in bubbles, stripe phases, and ringlike configurations, or combinations of both of them. Detailed phase diagrams are presented for systems consisting of $N=2$ up to $N=6$ particles. General rules are derived for the different subsequent transitions between those configurations and how the ground state configuration of a large system can be deduced from the one of a small number of particles.
\end{abstract}

DOI: 10.1103/PhysRevE.71.066204 PACS number(s): 05.45. $-\mathrm{a}, 45.20 .-\mathrm{d}, 45.05 .+\mathrm{x}, 61.46 .+\mathrm{w}$

\section{INTRODUCTION}

Competing attractive and repulsive interactions generate domain patterns in a wide variety of two- and threedimensional systems in nature $[1,2]$. In biology, liquid regions of biological membranes are formed by lipid bilayers and membrane proteins. The width of the liquid domains depends on the competition between line tension and electrostatic dipolar repulsion. Theory predicts an exponential scaling of this width as is confirmed by experiments [3]. In magnetic materials the domain structure with alternating spin orientation originates from the competition between shortrange exchange interaction and long-rang dipole interaction [4]. In antiferromagnetic materials, or layered transition metal oxides, holes interact through a competing long-range and short-range potential $[5,6]$. These competitions generate charge ordering and have possible relations to the mechanism of high-temperature superconductivity in doped cuprates [7] and bismuthates [8]. In materials science experiments with colloidal systems, combined with theoretical predictions, may lead to the design of novel soft materials and to a deeper understanding of the glass and gel states of matter [9]. Another example are the dispersion properties of chemical synthesized cobalt nanoparticles which were studied experimentally in Ref. [10]. Further experimental realizations of systems with competing interactions are the dynamic self-assembly of rings of charged metallic spheres [11,12], pattern formation in dynamical systems [13] and crystallization and aggregation in a colloid-polymer suspension [14].

Recently, it became clear that the pattern formation in the above systems displays common structural features. These common features, like the spatial modulation, suggests a universal approach for explaining the behavior of these systems. In recent molecular dynamics simulations [15-18] simplified models with different types of interaction potential were performed and lead to a good agreement with the experimental

\footnotetext{
*Electronic address: kwinten.nelissen@ua.ac.be

†Electronic address: bart.partoens@ua.ac.be

*Electronic address: francois.peeters@ua.ac.be
}

observed patterns. The goal of these simulations was to control these self-organized patterns by adjusting a small number of physical parameters. This is important in order to understand these self-assembled systems and to control the size, orientation, and structure of the formed patterns. Notice that all theoretical studies until now were done for infinite systems.

In our modern age of nanoscience, the study of finite size effects has become very important. Often the nanosystem is put on a surface and motion is limited in two dimensions (2D). This motivated us to apply an extra confinement potential to a 2D system and study the effect of a boundary on the patterns generated by the competing interactions. The system under study in the present paper is composed of a finite number of classical particles which interact through a repulsive Coulomb potential and an attractive exponential potential. The particles move in a $2 \mathrm{D}$ plane where the motion is confined by a parabolic potential. The competition between the short-range attraction and the long-range repulsion potential will generate a spontaneous spatial modulation of the particles. We found that the particles will organize themselves in various different phases like bubble phases (particles are organized in different bubbles), stripe phases (particles are organized in different lines on a ring), rings (particles are organized in different rings around the center) or combination of those phases depending on the strength and interaction range of the short-range potential, the strength of the confinement potential, and the number of particles.

The paper is organized as follows. In Sec. II our model system is introduced. The results for a small system are presented in Sec. III, those for intermediate systems in Sec. IV, and for large systems in Sec. V. Our conclusions are presented in Sec. VI.

\section{MODEL SYSTEM}

With our model system we are interested to study the effect of the competition of a short range attraction and a long range repulsion on the configurations in a finite system. We considered a system with $N$ pointlike classical particles 
interacting through a repulsive Coulomb potential and an attractive exponential potential as in Refs. $[15,16]$. The particles move in two dimensions where they are confined by a parabolic potential trap centered at the origin. The general dimensionless Hamiltonian of the system is written as

$$
H=\sum_{i=1}^{N} r_{i}^{2}+\sum_{i<j}^{N}\left(\frac{1}{\left|\mathbf{r}_{i}-\mathbf{r}_{j}\right|}-B e^{-\kappa \mid \mathbf{r}_{i}-\mathbf{r}_{j}}\right),
$$

using the following units: $r^{\prime}=\left(2 q^{2} / m \epsilon \omega_{0}^{2}\right)^{1 / 2}$ for the length, $E^{\prime}=m \omega_{0}^{2} r_{0}^{2} / 2$ for the energy with $m$ the mass of the particles and $\omega^{\prime}=\omega_{0} / \sqrt{2}$ for the frequency with $\omega_{0}$ the strength of the confinement potential. Note that the strength of the confinement potential in combination with the strength of the Coulomb repulsive part of the interparticle interaction determine the length and energy scale. The first term is the confinement potential, the second the repulsive part of the interparticle potential and the last the attractive part. The exponential term is determined by two parameters $B$ and $\kappa$, where $B$ determines the strength and $\kappa$ the interaction range of the attractive part of the interparticle potential. Notice that the repulsive part is long range while the attractive part is short range. But in the present work the ranges of the potentials should be compared with the finite size of our system.

To find the minimum energy configurations we used the Monte Carlo simulation technique extended with a Newton optimization technique [19]. This last technique increases the convergence and the accuracy of the obtained local minima. In order to be sure to have found the ground state configuration, we run the Monte Carlo simulation routine many times, starting with different random configurations. To assume that the obtained configuration is a stable one, the eigenvalues of the dynamical matrix, which are the squared eigenfrequencies of the system, are calculated and we required that they are positive. A negative eigenvalue implies that the obtained extremum corresponds to a local energy maximum or a saddle point.

\section{SMALL NUMBER OF PARTICLES}

In the following, phase diagrams for the configuration of small clusters are presented as a function of the interparticle potential parameters. In order to understand these phase diagrams better, it is useful to investigate the interaction potential first. The curves in Fig. 1 show the position $r$ of the extrema of the interaction potential between two particles for different $\kappa$ values as function of $B$. Each curve corresponds with two extrema, a minimum and a maximum, which join together at some minimum value of $B$. At this point the extrema disappears. For $B$ values below this point the attractive part of the potential becomes too small to compete with the repulsive part and the interparticle potential is purely repulsive. The distance between both extrema is inversely proportional to $\kappa$. Notice that the qualitative behavior is similar for low and high $\kappa$. For $\kappa=0$, the range of the attractive part of the potential becomes independent of the distance between the particles and does not affect the structure of the system. In the other limit, if $\kappa$ is very large, the range of the attractive potential becomes too short and will be unable to affect the

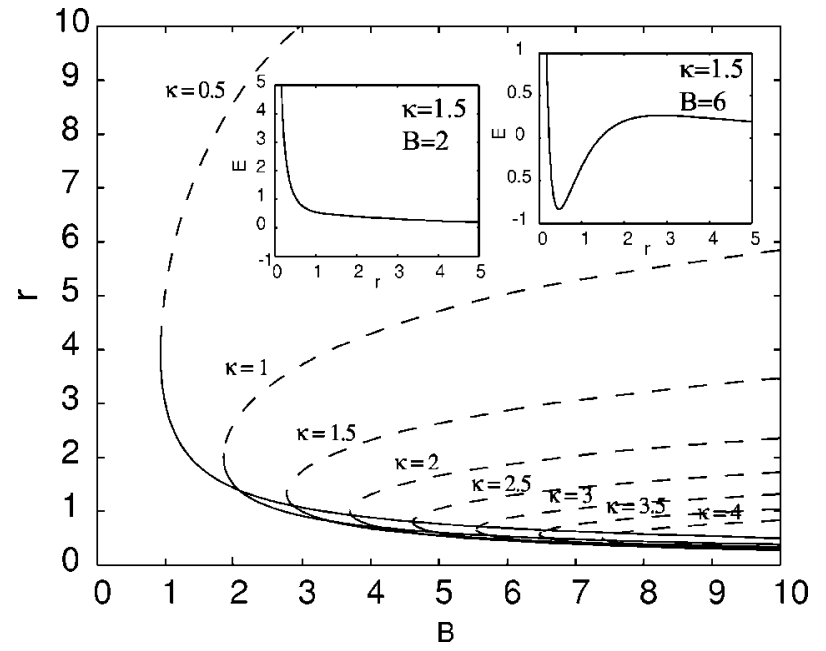

FIG. 1. Position of the extrema of the interparticle potential as function of $B$ for different $\kappa$ values. The full curves correspond to a minimum and the dashed curves to a maximum. The insets show the potential energy as function of the distance between the particles for $B=2$ and $B=6$ with $\kappa=1.5$.

structure of the system either. So one can expect a transition region in $(\kappa, B)$ space, where the attractive potential strongly competes with the repulsive Coulomb potential resulting in transitions between different particle configurations. Also one can expect that these transitions will appear around the same values in $(\kappa, B)$ for different number of particles confined in the parabolic trap. The reason is that the transitions are determined by the mean interparticle distance and this mean interparticle distance is approximately independent of the number of particles in a parabolic trap.

The configurations were studied as function of $\kappa$ and $B$. The results for the ground state configurations are summarized in phase diagrams for $N=2, \ldots, 6$ particles. In order to label the configurations, we use the following notation $\left\{n(k)^{s, b} ; m(l, d)\right\}$, where the numbers between brackets, $k, l$ and $d$, give the number of particles per shell in a stripe or bubble and $n$ and $m$ are the number of bubbles or stripes with the same configuration. The letters $s$ and $b$ stand respectively for the stripe and the bubble configuration. For example, the notation $\left\{2(2)^{s} ; 2(3)^{b}\right\}$ in Fig. 15 means that there are 2 stripes of two particles and 2 bubbles of 3 particles.

Let us first discuss the result for $N=2$, presented in Fig. 2 . From the analysis of the interparticle potential we know that when $B$ is sufficiently large, the interparticle potential has a minimum and a maximum, and consequently two configurations are possible: one in which the particles are close to each other and one in which they repel each other. The latter is only stable because of the presence of the confinement potential. The former we call the high $(H)$ and the latter the low $(L)$ density ring configuration, notated as $(2)^{H}$ and $(2)^{L}$, respectively. The first order phase boundary between both phases is given by the solid curve in Fig. 2, which to a good approximation can be represented by the line $B=2.9 \kappa-5.0$. This line ends in a critical second order transition point $(\kappa, B)=(5,8.6)$. Notice that for low $B$ values one can go continuously from one configuration to the other. 


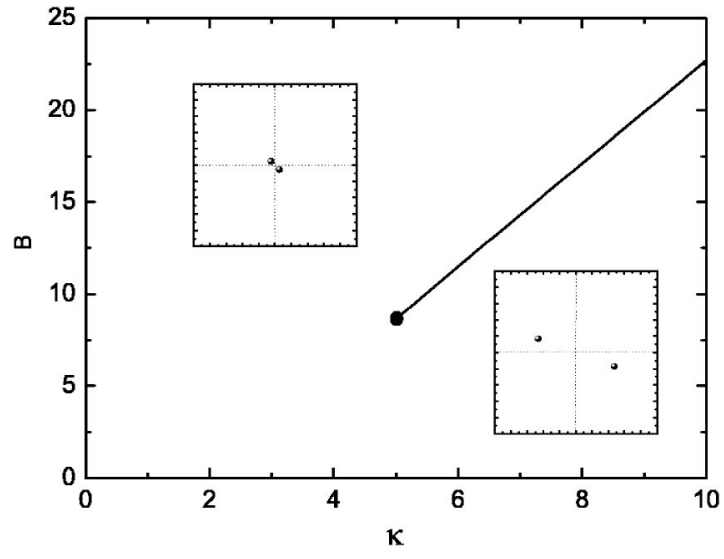

FIG. 2. Phase diagram for the ground state configurations of a classical cluster with $N=2$ particles. The insets shows the typical configurations in each region of the phase diagram.

In Fig. 3 we investigate a 3-particle system. The particles are arranged on a ring with different densities on both sides of the transition line, i.e., the $(3)^{L}$ and the $(3)^{H}$ configuration. The thin lines in Fig. 3 are equiradius lines for the ring configuration. The configuration in the low density region is determined by the Coulomb repulsive part of the interparticle interaction as found in previous simulations [20]. The configuration in the high density region is determined by the competition of the attractive and repulsive part of the interparticle interaction. The boundary between the low and high density configuration regions is a triple line (to a good approximation given by $B=2.80 \kappa-5.40)$ which ends in a tricritical point $(\kappa, B)=(4.1,6.8)$, below which no transitions occur. Exactly on the triple line, the $\left\{(1),(2)^{b}\right\}$-bubble configuration coexist with both ring configurations, as shown in Fig. 4. For this $\kappa$ value and $B$ range, 3 stable configurations are found which go through a single point on the triple line. In the bubble configuration on the triple line, two particles

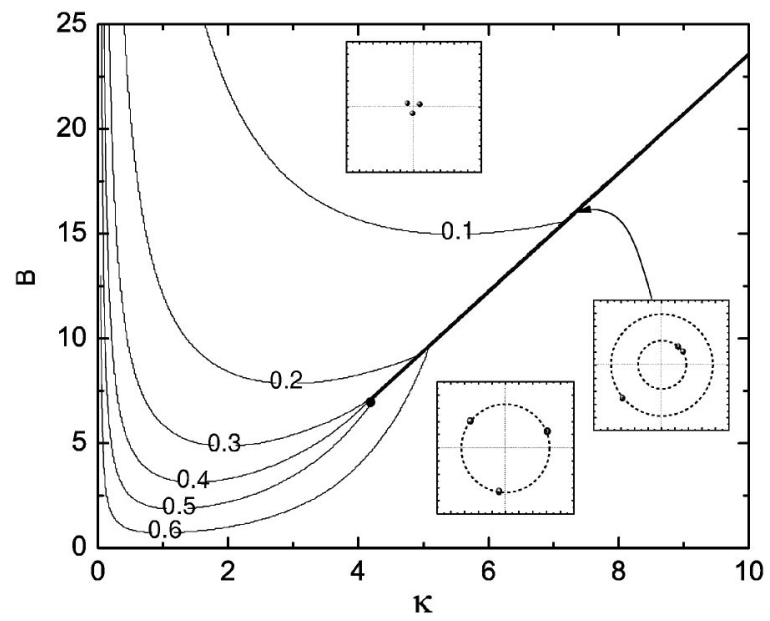

FIG. 3. Phase diagram for the ground state configurations of a classical cluster with $N=3$ particles. The insets show typical configurations in each region of the phase diagram. At the phase boundary three phases coexist and is therefore a triple line which ends up in a tri-critical point. The thin lines are equiradius lines for the ring configuration.

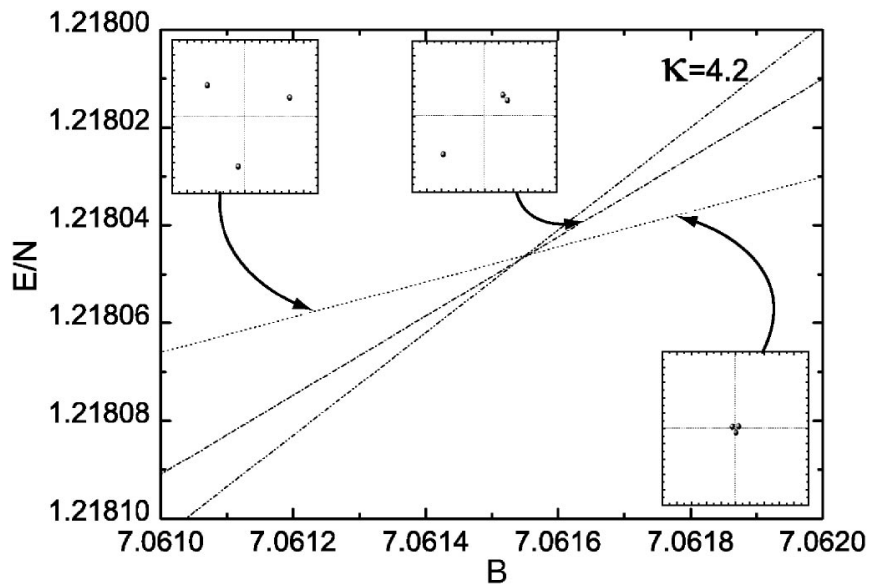

FIG. 4. The energy of all stable configurations, of a classical cluster with $N=3$ particles, as function of the amplitude $B$ of the attractive potential.

are grouped in a bubble and separated from the third particle by the Coulomb potential. Figure 5 shows the distance between two particles in the bubble and the distance between the bubble and a third particle as function of $\kappa$ following the bubble configuration on the triple line until the tri-critical point is reached. If $\kappa$ increases following the transition line, the distance between bubble and particle become equal to the distance between a particle and another effective defect particle with double charge and mass. This limit is plotted in Fig. 5 and is equal to $D=1.145$. In the other limit if $\kappa$ decreases and goes to the tri-critical point, the distance between the particles in the bubble and between the particle and the bubble become equal or in other words the bubble configuration becomes equal to the ring configuration. One can conclude that the triple line does not end up in a triple point where the high and low density ring configuration coexist with the bubble phase. Instead, all those phases become identical. In the eigenfrequency and radius plot in Fig. 6 for $B=8$ we see that the transitions on the triple line are of first order. Following the transition region to smaller $B$ and $\kappa$

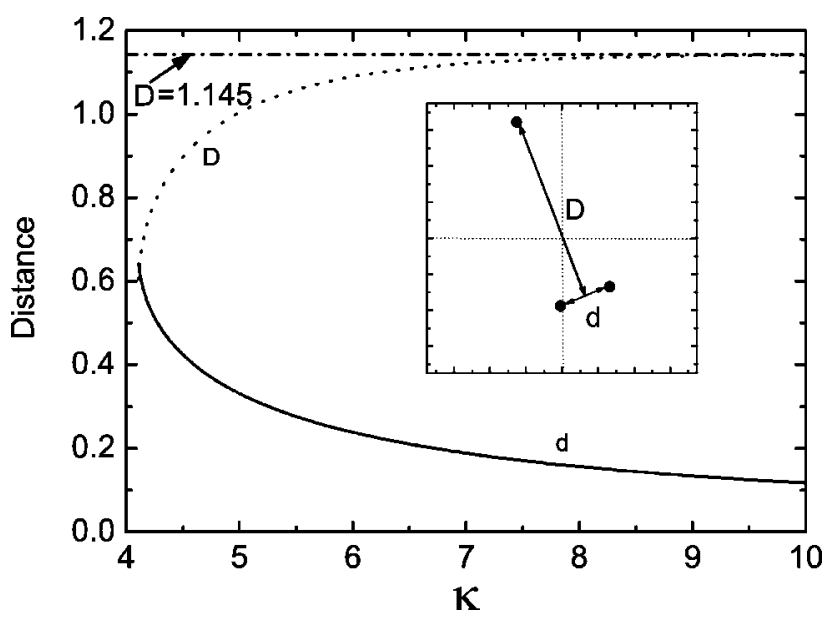

FIG. 5. The distance between the 2-particle stripe and a third particle and between the particles in the stripe following the transition line from high $\kappa$ to the tri-critical point. 


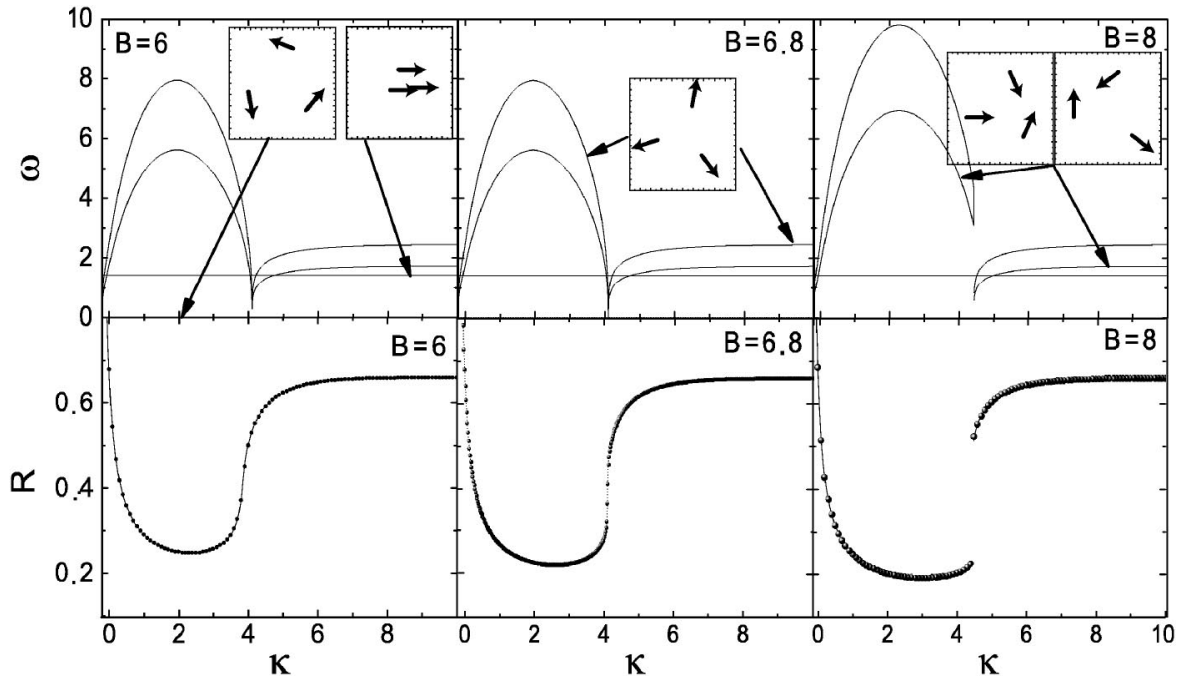

FIG. 6. Eigenfrequencies (upper figures) and distance of each particle to the center of the confinement potential (lower figures) of a 3-particle system as function of $\kappa$ for three different values of $B$. The insets show the corresponding eigenmodes. The center of mass mode and the one shown in the inset of the right figure are twofold degenerate. The curves for $B=6.8$ are going through the tri-critical point. values, we found that the lowest nonzero eigenfrequency goes to zero at the critical point indicating a second order transition as shown in Fig. 6 for $B=6.8$, which shows the eigenfrequencies for $B$ values near the critical point. Note that not a single but two frequency branches become zero indicating that the second order derivative in the tri-critical point is zero in more than one direction, which is consistent with the fact that the 3 configurations become the same in this point. Decreasing $\kappa$ and $B$ further leads to an increase in the lowest nonzero eigenfrequency and no transitions occur. In this region one can go in a continuous way from the $(3)^{L}$ configuration to the $(3)^{H}$ configuration. The radii in Fig. 6 for $B=6$ and equiradii lines in Fig. 3 illustrate the phase transition before and after this tri-critical point. The positions of the particles exhibit a jump at the first order transition. Notice also that the frequency of the breathing mode, the mode where all particles vibrate simultaneously in the radial direction (see insets of Fig. 6), becomes large in the high density region due to the strong mutual interaction.

In the 4-particle system (see Fig. 7) we have a similar high and low density region in which the particles are located on a ring. But the transition region is much richer, due to the fact that we can combine different number of particles in bubbles of different sizes. The first order phase boundaries are given to a good approximation by $B=2.7 \kappa-4.3, B$ $=2.8 \kappa-4.6$ and $B=3.0 \kappa-5.0$ (the second expression represents two transition lines which are very close to each other, see the circular inset in Fig. 7), which join together in a critical point at $(\kappa, B)=(4.0,6.6)$. Just like for the bubble phase in the 3-particle system, the 3 bubble phases of the 4-particle system can transform continuously (through a second order transition) into the same ring configuration. As a consequence all the phase boundaries must join in a single point, leading to the remarkable feature that in this point 4 phases become the same. The inclination of the lowest phase boundary is almost the same as for the 3-particle system. This behavior can be understood as follows: the formation of a two particle bubble, by increasing $B$ or decreasing $\kappa$, is independent of the number of particles but is determined by the interaction between two particles in a bubble. At high $\kappa$ values the system is in the low density ring configuration.
The interaction range of the attractive potential is too small to compete with the Coulomb potential. The system behaves as interacting through a Coulomb repulsion and trapped in a parabolic confinement potential. By decreasing $\kappa$, the shortrange potential becomes strong enough to compete with the Coulomb potential and bubble configurations are possible. The bubble configuration, with two particles grouped into a bubble, becomes the ground state by further decreasing $\kappa$. Such a bubble can be viewed as an effective defect particle with multiple charge and mass as was studied in Refs. $[21,22]$. Therefore the distance between the two other particles increases slightly and prevents them to group into another bubble. This process continues until all particles are grouped into a single bubble, i.e., the high density $(4)^{H}$ state. Notice that the phase diagram only shows the ground state configurations, but several metastable configurations can exist at the same time. These configurations are illustrated in Fig. 8, where the energy of the different stable states are plotted as function of $\kappa$ for fixed $B$. Notice that the state $\left\{2(2)^{b}\right\}$ becomes the ground state only over a very small $\kappa$

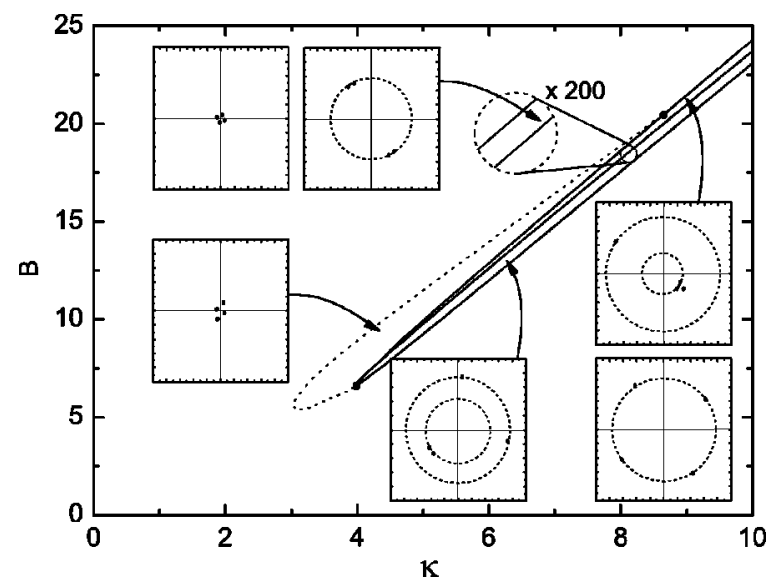

FIG. 7. Phase diagram for the ground state configurations of a classical cluster with $N=4$ particles. The straight lines indicate the first order transitions and the dashed line the second order transition. The insets depict typical configurations in each region of the phase diagram. 


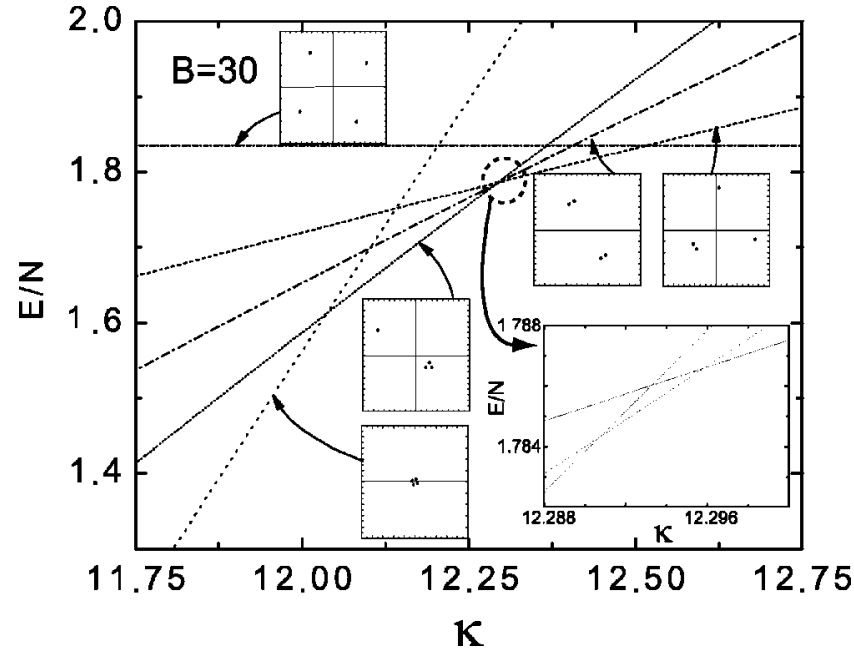

FIG. 8. The energy of all stable configurations, of a classical cluster with $N=4$ particles, as function of the interaction range $\kappa$ of the attractive potential for $B=30$.

region which is shown in one of the insets of Fig. 8. This is in contrast with a three particle system where we found a triple line where three phases coexist.

In a 4-particle system, the critical point, i.e., the second order phase transition, is extended from a single point to a line in the high density region, which stops in the critical point $(B, \kappa)=(20.4,8.6)$. In the $4<\kappa<8.5$ region this line is closely approximates by the line $B=2.4 \kappa-0.8$. This line is absent in the 3-particle system because this system forms a Wigner lattice in the ground state which is proven to be very stable. In a 4-particle system, the particles form a ring and the triangular structure is lost. These particles on the ring are more free to move in comparison with particles in a triangular structure and this causes the system to undergo, in the high density configuration, a second order transition by grouping two opposite particles in the center of the parabolic confinement to minimize the energy. Figure 9 shows that the first nonzero eigenfrequency goes to zero at the second order transition. The other transitions occur through first order transitions.

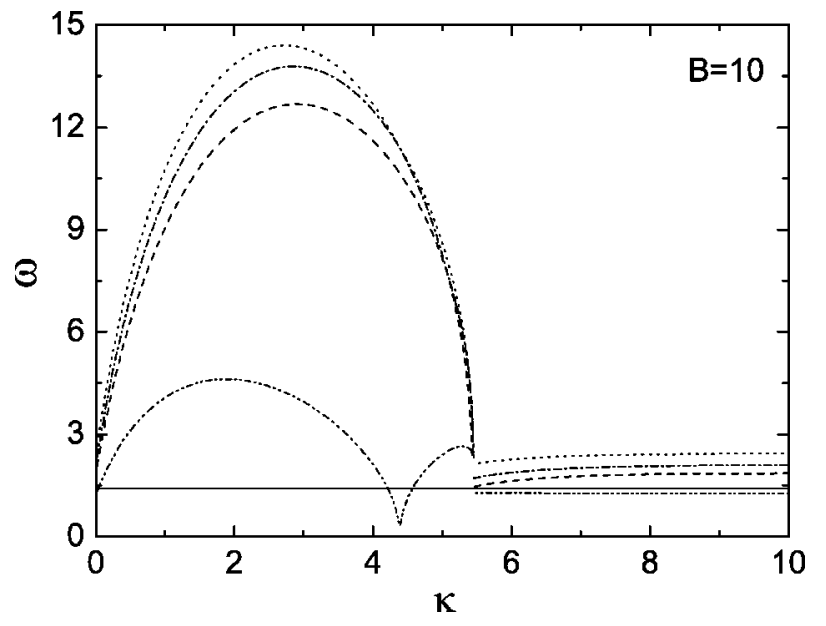

FIG. 9. The nonzero eigenfrequencies for a 4-particle system, as function of the interaction range $\kappa$ with $B=10$.

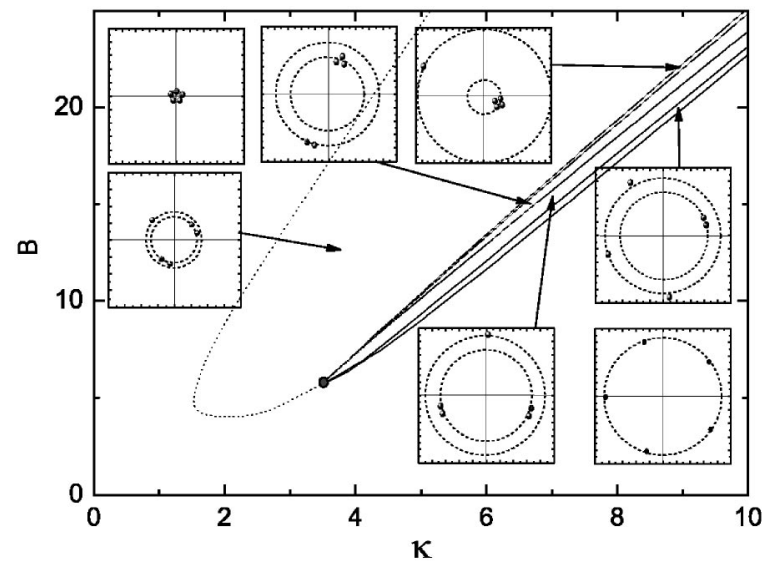

FIG. 10. Phase diagram for the ground state configurations of a classical cluster with $N=5$ particles. The straight line shows the first order transitions and the dashed lines the second order transition. The insets show the typical configurations in each region of the phase diagram.

In a 5-particle system there is again a transition region which separates a high and low density ring configuration with a second order transition line in the high density ring configuration. The first order phase boundaries are given to a good approximation by $B=2.6 \kappa-3.4, B=2.7 \kappa-3.6, B$ $=2.8 \kappa-4.0, B=2.9 \kappa-4.5$, and $B=2.9 \kappa-4.7$ which join together in a critical point at $(\kappa, B)=(3.5,5.7)$. The second order transition line in the region $2<\kappa<10$ is closely approximated by the line $B=2.9 \kappa-0.7$. In the critical point this second order line joins the first order line similar to what was found for the 4-particle system (see Fig. 7). Making the comparison between the 4- and 5-particle phase diagrams, the inclination of the second order transition line in the phase diagram is increased and the first order transition region is extended to smaller $\kappa$-values. The first order transition region is extended due to the increased number of possible configurations which is proportional to the number of particles squared. The inclination of the second order transition line is increased due to the increasing diameter of the ring. This increasing diameter results in a weaker confinement of the particles on the ring and allows a second order transition at lower $\kappa$-values. Notice that all phase boundaries join together in a single critical point $(\kappa, B)=(3.5,5.7)$. We can understand this as follows, if we follow the phase transition lines for decreasing $\kappa$, the particle configurations resemble more and more the (5)-ring configuration (this behavior is similar to the one of a 3-particle system as seen in Fig. 6) which is the configuration at the critical point. The different phases in Fig. 10 can be easily understood intuitively as an increased clustering of the particles and finally for small $\kappa$ a collapse into a single bubble. The energies of all the states are given as function of $\kappa$ in Fig. 11. Notice that from all of the 9 stable configurations only 6 are realized as ground state configurations.

The phase diagram for the $N=6$ system (see Fig. 12) is qualitatively different from the previous cases. There is only a short second order transition line, which at a first glance is unexpected. In comparison with previous systems, there is one particle in the center of the configuration preventing a 


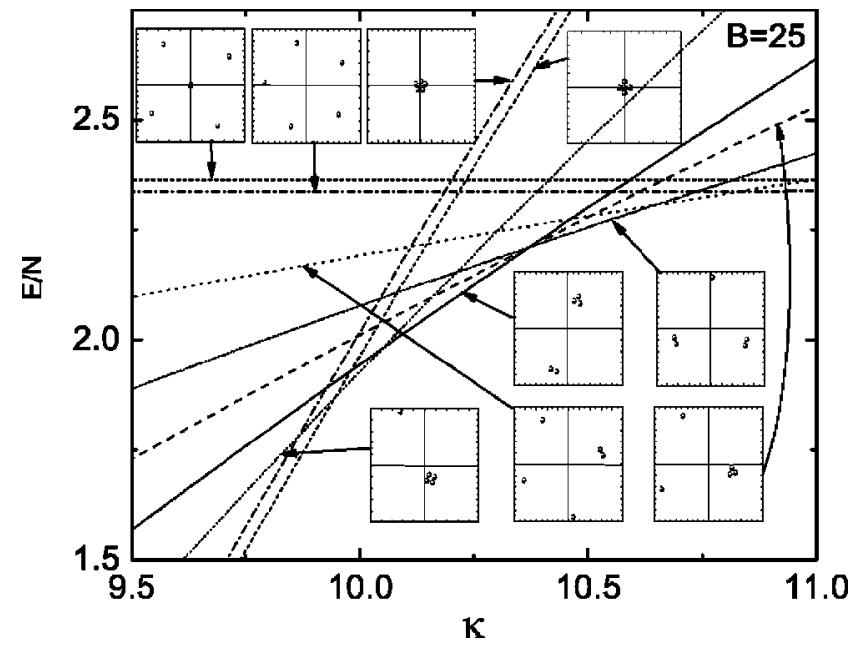

FIG. 11. The energy of all stable configurations, of a classical cluster with 5 particles, as function of the interaction range $\kappa$ of the attractive potential.

second order transition in the ground state configuration $(1,5)^{H}$. However, the ring configuration (6), without a particle in the center (which is the ground state in a small region), can go through a second order phase transition in which the particles group themselves in bubbles of two. The ground state configuration for a very large value of $\kappa$ is the $(1,5)^{L}$ configuration. The first order phase boundaries are given to a good approximation by $B=3.2 \kappa-6.3, B=2.9 \kappa$ $-5.3, B=2.2 \kappa-3.0$ (representing two phase lines) and $B$ $=2.5 \kappa-2.6$ (representing two phase lines). In contrast to the previous systems, one can see two critical second order joining points for the first order phase transition lines at $(\kappa, B)$ $=(3.5,6.3)$ and $(\kappa, B)=(4.0,6.5)$. This is because also the $(6)$ configuration exists as a ground state in a small region around the joining points of the phase transition lines. Therefore, if one follows the phase transition region for decreasing $\kappa$, the configuration can go in a continuous way to the $(1,5)$ or the $(6)^{H}$ configuration, leading to the critical point. One can see that the most circulatory configurations go to the (6)

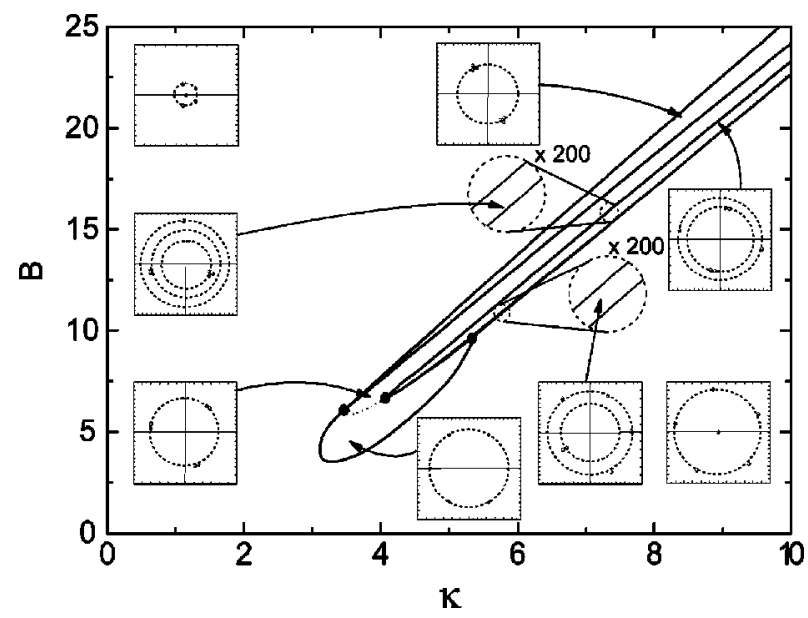

FIG. 12. Phase diagram for the ground state configurations of a classical cluster with $N=6$ particles. The insets show the typical configurations in each region of the phase diagram.
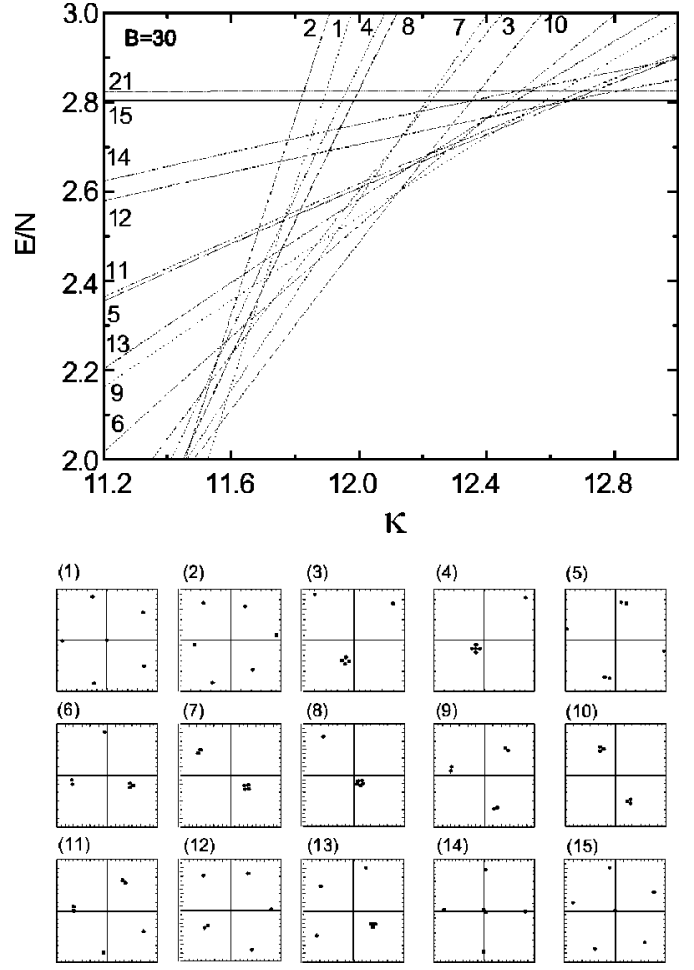

FIG. 13. The energy of all stable configurations, of a classical cluster with 6 particles, as function of the interaction range $\kappa$ of the attractive potential. The corresponding configurations are depicted in the lower figures.

configuration and the other to the $(1,5)$ configuration. Finally in Fig. 13 the energies of all the possible stable configurations are plotted as function of $\kappa$. With increasing $\kappa$ we found the following ground states: 1, 2, 5, 6, 9, 10, 12 and 15 , where the number corresponds to the curves in the upper figure of Fig. 13 and the corresponding configurations are shown in the lower figures of Fig. 13.

\section{INTERMEDIATE NUMBER OF PARTICLES}

In this section we want to see if we can infer the behavior of larger systems from our knowledge of the few particle systems of the previous section. Therefore, we will take a closer look to the 10-, 20- and 30-particle systems. In the previous section we found that the number of possible stable states increases very rapidly with increasing number of particles, e.g., 9 in Fig. 11 for $N=5$ and 21 in Fig. 13 for $N$ $=6$. There is also a corresponding increase of the number of possible phases as ground state. Therefore, for systems with more particles it becomes almost impossible to construct a detailed phase diagram. To obtain still information about the influence of changing $B$ on the system we make a cross section in $(\kappa, B)$ space at $\kappa=4$.

In this paragraph the influence of changing $B$ on the ground state of a 10-particle system is studied and $\kappa$ is fixed at 4. In Fig. 14, the number of stable states, the energy differences of the metastable states with the ground state, the distance of the particles of the ground state configuration to the center of the confinement potential, and the eigenfre- 


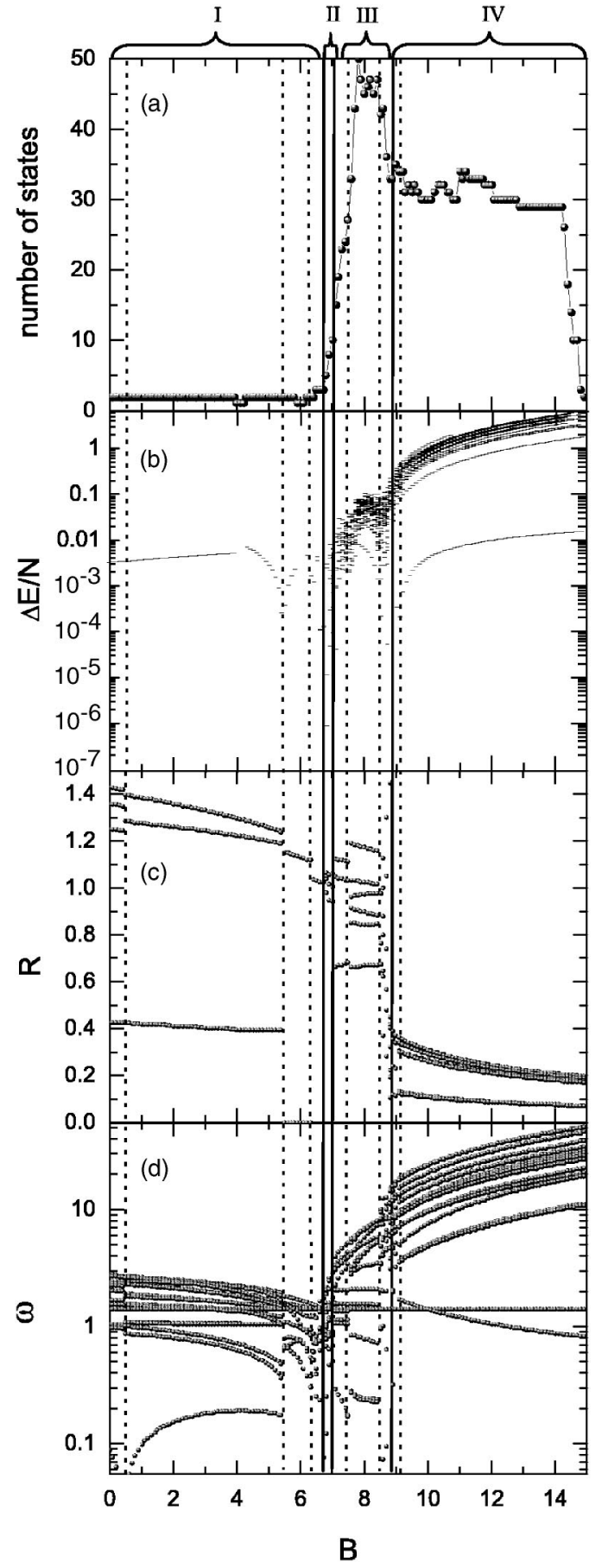

FIG. 14. (a) Number of stable states, (b) energy difference of the metastable states with respect to the ground state, (c) the distance of the particles to the center, and (d) eigenfrequencies of the ground state of a 10-particle system as function of $B$ for $\kappa=4$. The vertical lines mark the different regions: I ring, II stripe, III bubble, and IV cluster phases. Subdivisions in a region are marked with a short dashed line.

quencies of the ground state configuration are given as function of $B$. We distinguished 4 main regions which are separated by the full vertical lines: region I corresponds to low density ring configurations, II to stripe configurations, III to bubble configurations, and IV to high density ring configu-
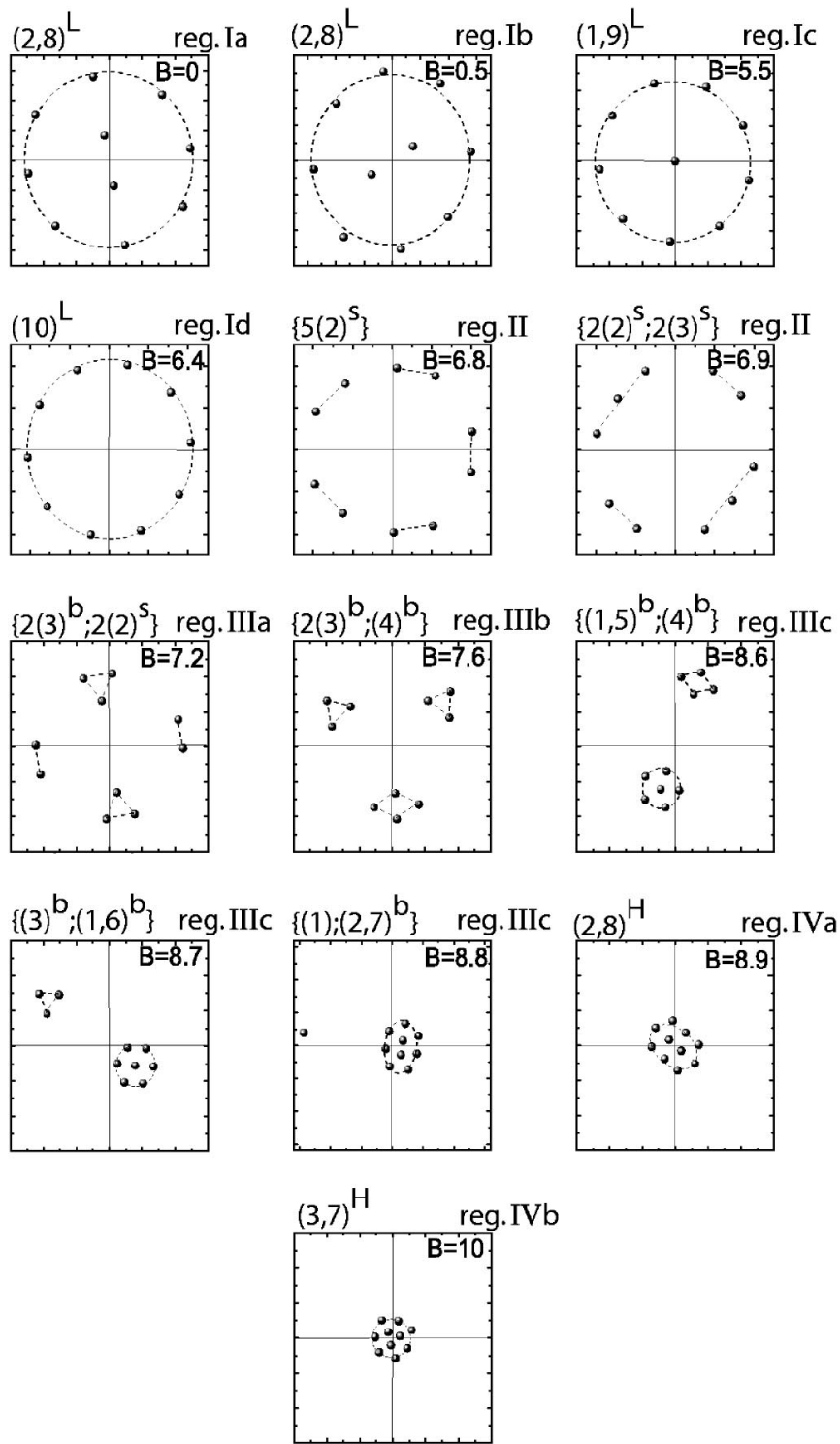

FIG. 15. Snapshots of the ground state configuration for a $N=10$-particle system with $\kappa=4$. The different regions refer to Fig. 14.

rations. The snapshots of Fig. 15 are labeled with the region number to which the configuration belongs. In region I the ground state configurations are ring configurations. Different ring configurations are found as function of $B$ and therefore region $\mathrm{I}$ is subdivided, marked by the short dashed lines in Fig. 14. For $B=0$ the $(2,8)^{L}$-ring configuration is the ground state. If one increases $B$, the system changes slightly by rotating the inner particles with respect to the other particles, through a second order transition. If one increases $B$ further, the particles redistribute themselves over different rings in order to form first the $(1,9)^{L}$ configuration and subsequently the $(10)^{L}$-ring configuration by promoting particles from the inner shell to the outer shell. Next, the $(10)^{L}$-ring configuration undergoes a second order transition and the particles group themselves in stripes of two particles on a ring (or pentagon) and form the $\left\{5(2)^{s}\right\}$-stripe configuration, immediately followed by another first order transition and form the 
$\left\{2(2)^{s}, 2(3)^{s}\right\}$-stripe configuration (in which the particles are grouped in stripes of 2 or 3 particles which are roughly situated on a rectangle). These stripe phases constitute region II. In region III bubble phases are formed: the particles in the two 3-particles stripes form two 3-particles bubbles resulting in the $\left\{2(2)^{s}, 2(3)^{b}\right\}$ configuration followed by a transition to the $\left\{2(3)^{b},(4)^{b}\right\}$ configuration. The system consists now of two bubbles of three particles and one bubble of four particles. This last configuration is very symmetric and is the ground state over a relative large $B$ range, indicated by the two dashed lines in Fig. 14 around $B=8$. Finally, if one increases the amplitude of the attractive potential further, the particles redistribute themselves over larger bubbles until all particles are grouped in a single bubble in region IV in order to form the $(2,8)^{H}$ deformed ringlike configuration. Also this high density ring configuration undergoes a first order transition, marked by a dashed line in Fig. 14, to the final ground state configuration $(3,7)^{H}$. Notice, that qualitatively the transitions occur through the same mechanism as found in smaller systems. Moreover, the clustering of the particles in bubbles occurs around similar values for the parameters of the attractive part of the interparticle potential as found in smaller system.

Constructing a complete phase diagram from the Monte Carlo simulations is very time consuming, as the number of stable configurations can be very large. This is illustrated in Fig. 14(a) for $N=10$ and $\kappa=4$ where $B$ is varied with steps of $\Delta B=0.1$. We preformed 50.000 trials and used the criterium that two states are taken to be different if $\Delta E=10^{-7}$. Around $B=8$ a peak appears which occurs in the bubble phase region III, where metastable states are easily formed by redistributing particles between the bubbles and rotating the bubbles with respect to each other. For small $B$ (region I) the number of stable states is small because the system is close to a normal Coulomb cluster. For larger values of $B$ (region IV), the total number of stable states stays large. Notice that the energy differences between the ground state and the metastable states [shown in Fig. 14(b)] become larger after region III. This implies that the stability of the metastable states decreases for increasing $B$ and the number of stable states decreases.

The same quantities as in Fig. 14 are shown in Fig. 16 but now for a 20-particle system. Note the increase, an order of magnitude, in the number of stable states for $B>7$ [Fig. 16(a) ] as compared to the $N=10$ case. Snapshots of configurations for different $B$ are shown in Fig. 17. As for $N=10$, we can distinguish four main types of configurations (I low density rings II stripes, III bubbles, and IV high density rings) are present and marked by full vertical lines in Fig. 16. Subdivisions are marked by short dashed lines. From both figures, one can see that the particles first redistribute themselves over different rings until the $(3,17)^{L}$-ring configuration in region I. Notice that in contrast to $N=10$, for $N=20$ two well developed rings are observed. Next in region II, the particles on the outer ring group themselves in stripes through a second order transition. Note that the stability of the stripe phase is reduced tremendously: in Fig. 16 it is marked by one vertical line. The only stripe phase in this region is the $\left\{(3) ; 2(3)^{S} ;(11)^{S}\right\}$ configuration. The outer par-

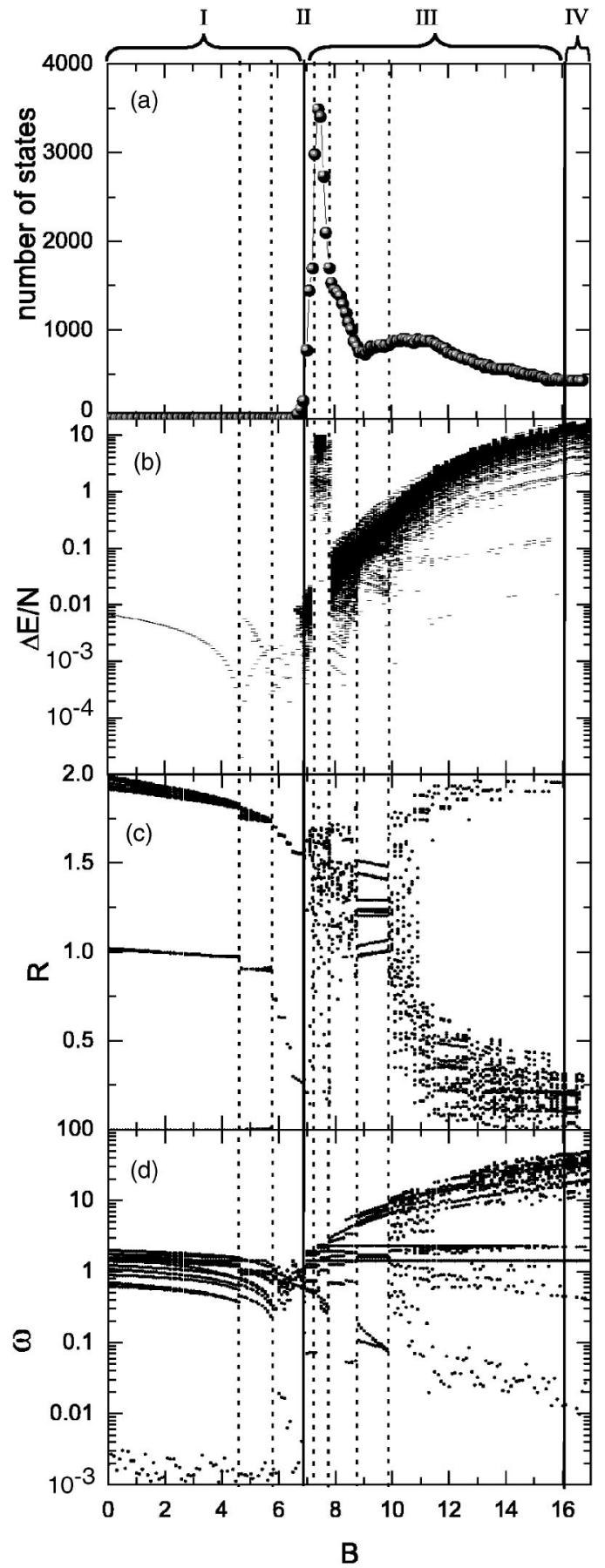

FIG. 16. (a) Number of stable states and (b) the distance of the particles to the center and (c) eigenfrequencies of the ground state of a 20-particle system as function of $B$ with $\kappa=4$. The vertical dashed lines mark different types of configurations in the ring and the bubble region.

ticles form almost a perfect ring, however a detailed investigation of the interparticle distances shows the existence of two curved stripes of 3 particles and one long curved stripe of 11 particles. By further increasing $B$, particles group in bubbles in region III through first order transitions. The width of this region has clearly increased in comparison with the 10-particle case. The chaotic behavior of the radii of the particles in region III [shown in Fig. 16(c)] indicates a fast 

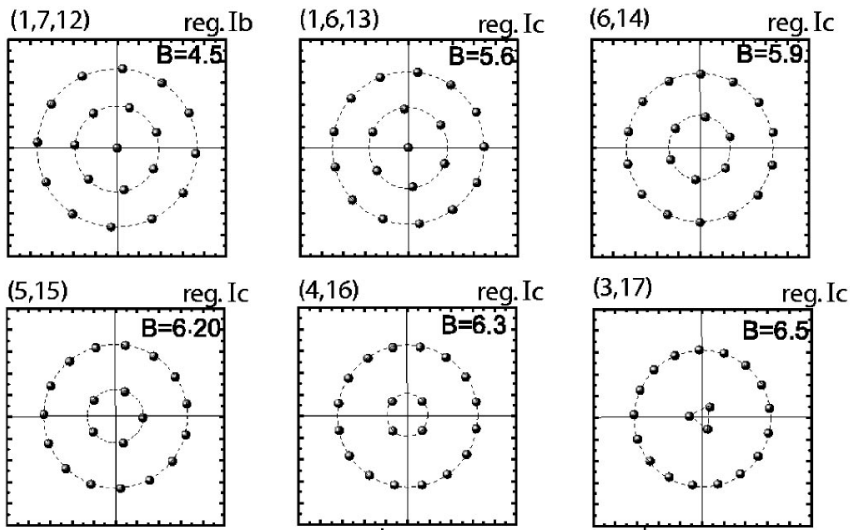

$\left\{(3) ; 2(3)^{\mathrm{s}} ;(11)^{\mathrm{s}}\right\}$ reg. II
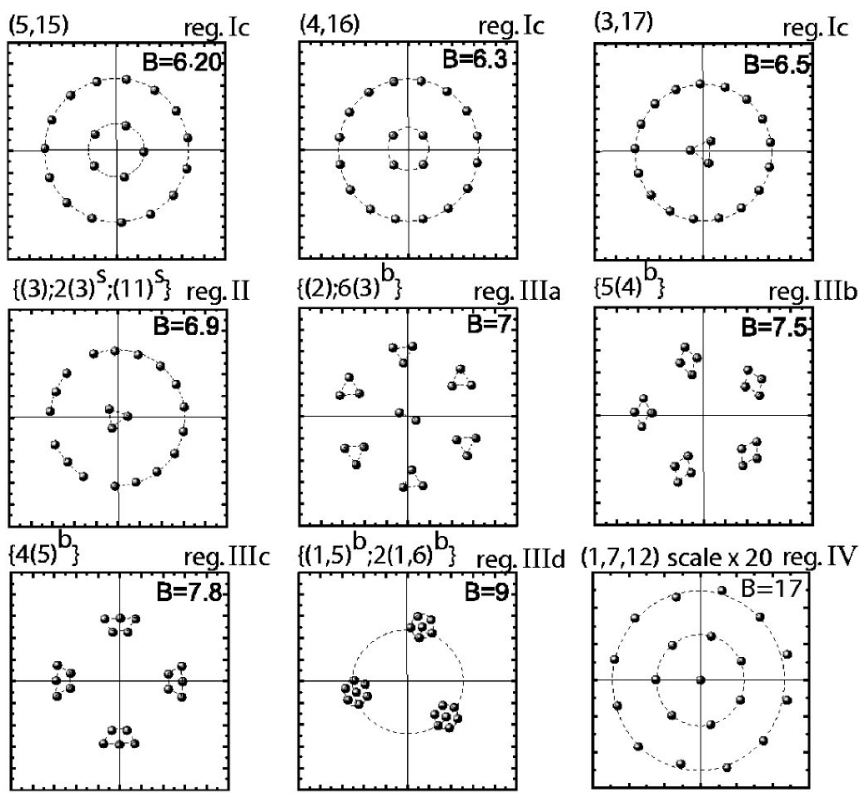

FIG. 17. Snapshots of the ground state configuration for a $N=20$-particle system with $\kappa=4$. The different regions refer to Fig. 16.

redistribution of the particles between the different bubbles with changing $B$. The number of particles with a radius smaller than 1 clearly increases for increasing $B$, which shows that the number of particles in the central bubble grows until all particles are grouped into the $(1,7,12)^{H}$-ring configuration in region IV. Only around $B=9$, a surprisingly stable configuration exists, which corresponds with the small dip in the number of stable configurations [see Fig. 16(a)]. This configuration is triangular and consists of 2 clusters of 7 particles and one with 6 particles (see Fig. 17). On the other hand, one can see from Fig. 16(b) that around $B=7.5$ the energy difference of the 5-particle bubbles configuration is rather large. If one looks at the eigenfrequencies one can see, for higher $B$, that the first nonzero eigenfrequency becomes larger. This means that the bonding between the particles becomes stiffer which can be explained by the higher particle density of the system.

For the 30-particle system we limit the discussion and show the distances of the particles to the center as a function of $B$ in Fig. 18 for $\kappa=4$. Figure 19 shows a few snapshots of ground state configurations for different $B$ values. Again the four regions are marked. Many more different low density ring configurations are found in region $\mathrm{I}$ : with increasing $B$ the particles jump from the inner rings to the outer ring. The stripe phase region II is again a very small region and on the scale of Fig. 18 is reduced to a single line. The stripe phase $\left\{(7)^{S} ;(13)\right\}$ around $B=6.7$ consists of a bent stripe in the center and a closed ring around it. The stability region of the

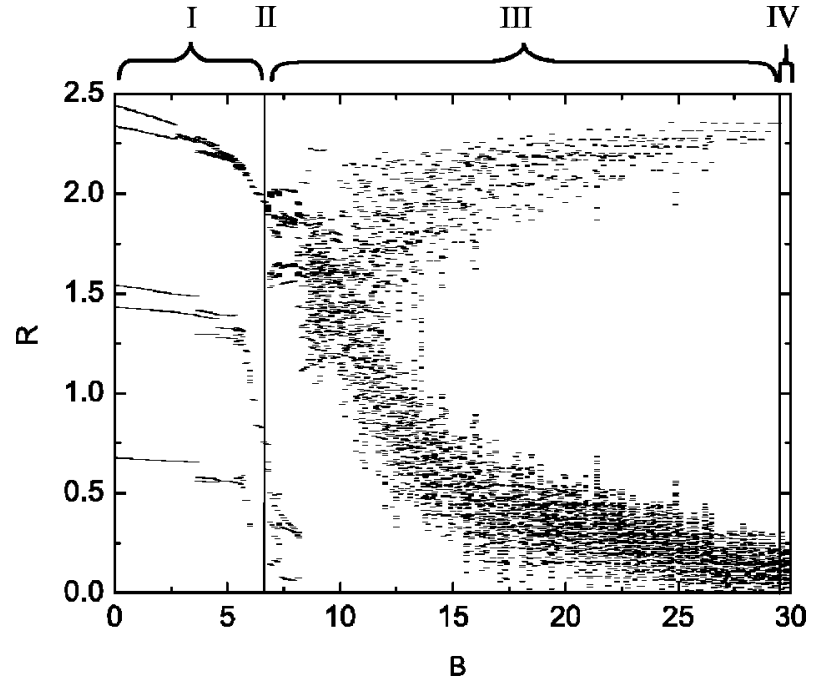

FIG. 18. The distance of the particles to the center of a 30particle system as function of $B$ with $\kappa=4$. The vertical dashed lines mark the redistribution of the particles over different rings $(B<6.7)$ and the bubble region $(6.7<B<29)$.

bubble phase region III now has even more increased, because it takes longer before all particles are grouped into the central bubble. Eventually, the high density ring configuration $(1,5,10,14)^{H}$ is reached around $B=29$.
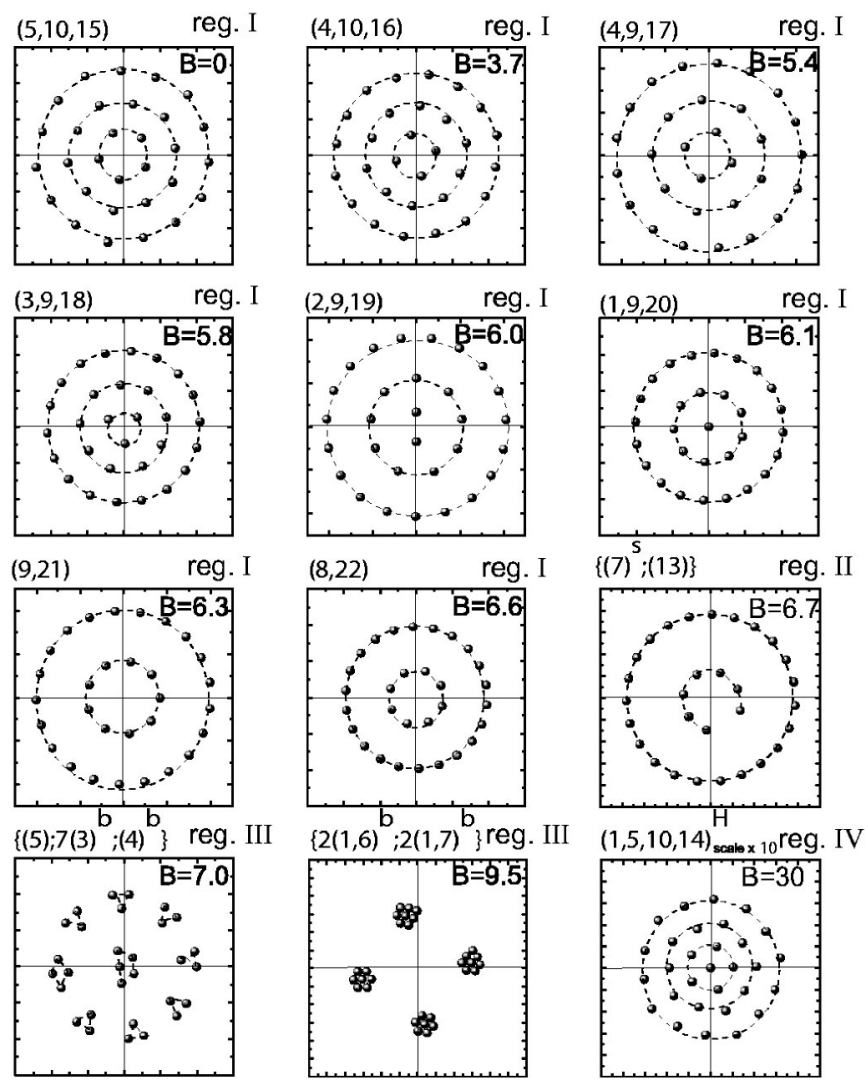

FIG. 19. Snapshots of the ground state configuration for a $N=30$-particle system with $\kappa=4$. 


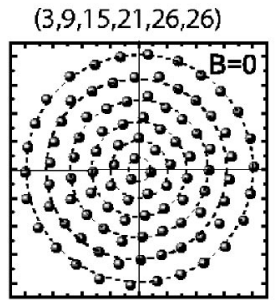

$(3,9,14,19,19,36)$

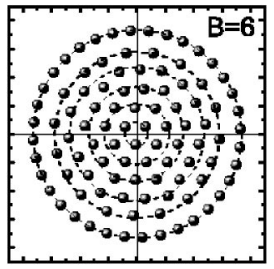

$(9,9,9,29,44)$

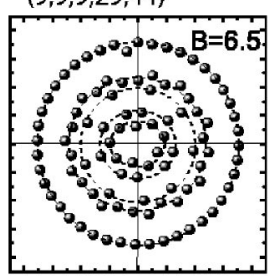

$\{2(2,6) ; 3(2,7) ;(2,8) ; 2(1,6) ;(3) ;(1,5) ;(1,7)\}$
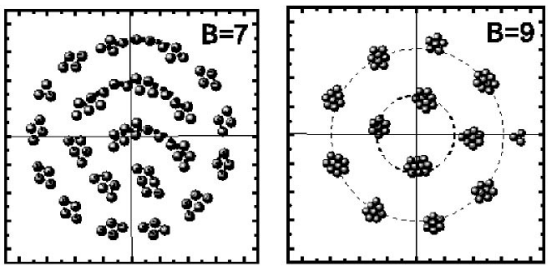

FIG. 20. Snapshots of configurations for a $N=100$-particle system with $\kappa=4$.

From the above it is clear that with increasing number of particles the stability region of the stripe phases decreases while it increases for the bubble phases.

\section{LARGE SYSTEMS}

The series $N=10,20,30$ already gives us an idea of the richness of the possible realizable configurations which are a consequence of the competition between the confinement potential and the repulsive and attractive parts of the interparticle interaction. To illustrate the increased complexity with $N$ we consider as a final example the 100-particle system. We show in Fig. 20 snapshots of ground state configurations for 5 different $B$ values with $\kappa$ fixed at 4 . For small $B$, we find again the low density ring configuration (note even the onset of the hexagonal structure in the center). Increasing $B$ leads to a clustering of different rings in bands, as shown in the configuration for $B=6.5$ in Fig. 21. With a further increase in $B$, these bands break up, resulting in a phase which is equivalent to the stripe phase (see the configuration for $B=7$ in Fig.

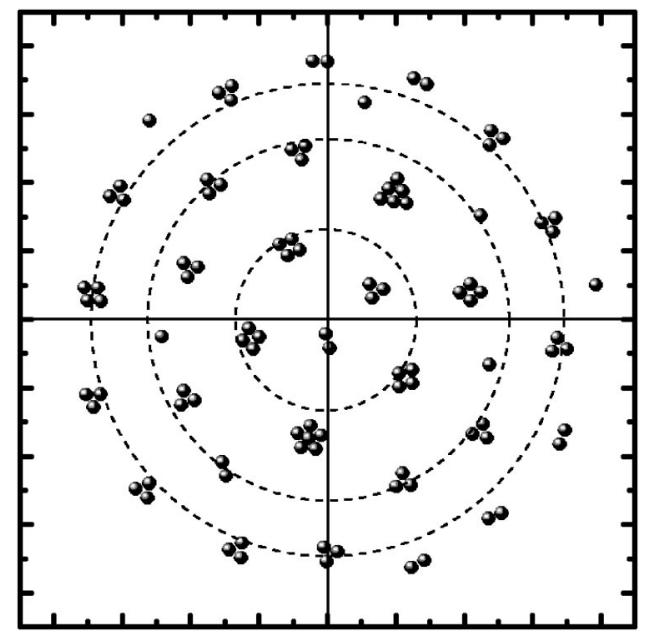

FIG. 21. Configuration for a $N=100$-particle system with $\kappa=6$ and $B=13.5$.
20). After the stripe phase, many different bubble phases are obtained.

From previous analysis of intermediate size and large systems we can formulate some general rules for different transitions between regions from the low to the high density configuration: (i) the particles redistribute to higher and less shells through first order transitions; (ii) the particles group in stripes located on shells through a second order transition; (iii) the particles in these stripes collapse into bubbles through first order transitions; (iv) the particles organize themselves in bubbles of different sizes and finally all particles are grouped in a single bubble through first order transitions. These transitions to bubbles occur for similar parameters of the attractive part of the inter-particle potential as found for smaller systems.

Finally, we want to investigate if we can predict even more properties of a large system with the obtained knowledge about smaller systems. As an example we want to predict for which $B$ value a 100 particle system consists mainly of 3-particle bubbles at $\kappa=6$. From the phase diagram for a 6-particle system (Fig. 12) we find that 3-particle bubbles are formed at $\kappa=6$ for $B=13.5$. The ground state configuration for 100 particles for the same parameters is shown in Fig. 21. The ground state configuration was obtained from 100 different trials. Note that the system is organized in small bubbles. These bubbles can be seen as effective particles with multiple charge and mass which interact through a Coulomb repulsive potential. These particles organize themselves in rings around the center of the central confinement potential. The average number of particles is found to be 2.77 particles per bubble which is very close to the predicted number of 3 particles per bubble using the phase diagram of a small system. This example shows that we can understand and predict some of the behaviors of larger systems starting from our knowledge of small systems. For larger systems, a bubble can be treated as an effective particle having multiple charge and mass. The subsequent multitype particle system is the finite analogon of the alloy problem.

\section{CONCLUSIONS}

In this paper we investigated a finite size system of classical charged particles with competing short-range attraction and a long-range repulsion potential. A rich variety of different configurations were obtained, the number of which increases with the number of particles. Transitions between the different configurations are centered around a strip located near $B \approx 2.5 \kappa-3.5$. This strip separates two identical ring configurations with different density, i.e., the low and high density ring configuration. The inclination of the first order phase boundary with smallest $B$ of this region does not depend on the number of particles in the system, but only on the individual interaction between the two particles. The width of the transition region is determined by the number of particles in the system because a deeper local potential minimum is needed to group all particles together in a single bubble. If one follows the ground state configuration starting from the low density configuration to the high density configuration through the transition region, we found that the 
particles are successively grouped into bubbles. This grouping is almost independent of the number of particles and occurs around the same parameters of the attractive part of the interparticle potential. The existence of a second order transition in this high density region depends on the structure of the configuration. It does not appear in configurations with a triangular arrangement of particles. For systems with more particles, the number of stable configurations grows very rapidly with $N$. With increasing the attractive part of the interaction potential, intermediate size and large systems all pass through four main phases: low density ring configurations, stripe phases, bubble phases and finally the high density ring configuration. The global qualitative behavior can be deduced from systems with a small number of particles for which we presented the full $(B, \kappa)$ phase diagram. The transition between a stripe phase and a bubble phase occurs through a first order transition. The transition between a ring configuration and the stripe phase occurs through a second order phase transition.

\section{ACKNOWLEDGMENTS}

This work was supported by the Flemish Science Foundation (FWO-Vl).
[1] M. Seul and D. Andelman, Science 267, 476 (1995).

[2] G. M. Whitesides and B. Grzybowski, Science 295, 2418 (2002).

[3] S. L. Keller and Harden M. McConnell, Phys. Rev. Lett. 82, 1602 (1999).

[4] C. Kittel, Phys. Rev. 70, 965 (1946).

[5] B. P. Stojkovic, Z. G. Yu, A. L. Chernyshev, A. R. Castro Neto, and N. Grønbech-Jensen, Phys. Rev. B 62, 4353 (1999).

[6] B. P. Stojkovic, Z. G. Yu, A. L. Chernyshev, A. R. Castro Neto, and Niels Grønbech-Jensen, Phys. Rev. Lett. 82, 4679 (2000).

[7] V. J. Emery, S. A. Kivelson, and P. V. Zachar, Phys. Rev. B 56, 6120 (1997).

[8] A. Aharony and E. A. Auerbach, Phys. Rev. Lett. 70, 1874 (1993).

[9] F. Sciortino, Nat. Mater. 1, 145 (2002).

[10] G. A. Held, G. Grinstein, H. Doyle, Souheng Sun, and C. B. Murray, Phys. Rev. B 64, 012408 (2001).

[11] B. A. Grwybowski, J. A. Wiles, and G. M. Whitesides, Nature (London) 405, 1033 (2003).
[12] B. A. Grwybowski, J. A. Wiles, and G. M. Whitesides, Phys. Rev. Lett. 90, 083903 (2003).

[13] J. P. Gullup and J. S. Langer, Rev. Mod. Phys. 71, 396 (1999).

[14] E. H. A. de Hoog, W. K. Kegel, A. van Blaanderen, and H. N. W. Lekkerkerker, Phys. Rev. E 64, 021407 (2001).

[15] C. J. O. Reichhardt, C. Reichhardt, I. Martin, and A. R. Bishop, Phys. Rev. Lett. 90, 026401 (2003).

[16] C. J. O. Reichhardt, C. Reichhardt, I. Martin, and A. R. Bishop, Phys. Rev. Lett. 92, 016801 (2004).

[17] G. Malescio and G. Pellicane, Nat. Mater. 2, 97 (2003).

[18] A. D. Stoycheva and S. J. Singer, Phys. Rev. Lett. 84, 4657 (2000); Phys. Rev. E 65, 036706 (2002).

[19] V. A. Schweigert and F. M. Peeters, Phys. Rev. B 51, 7700 (1995).

[20] V. M. Bedanov and F. M. Peeters, Phys. Rev. B 49, 2667 (1994).

[21] K. Nelissen, B. Partoens, and F. M. Peeters, Phys. Rev. E 69, 046605 (2004).

[22] M. Kong, B. Partoens, and F. M. Peeters, Phys. Rev. E 65, 046602 (2002). 